\title{
Diferencias en las Actitudes Frente a la Deshonestidad Académica entre Estudiantes de Pregrado de Administración y de Economía en la provincia del Guayas, Ecuador
}

\author{
Danny Arévalo-Avecillas*, Carmen Padilla-Lozano, José Perez-Villamar y Manuel Matute-Fernández \\ Universidad Católica de Santiago de Guayaquil, Km 1/2 vía Carlos Julio Arosemena, Guayaquil, Ecuador. \\ (e-mail: econ.darevalo@gmail.com; carmen.padilla@cu.ucsg.edu.ec; jose.perez@cu.ucsg.edu.ec; \\ manuel.matute.fernandez@gmail.com)
}

* Autor a quien se debe ser dirigida la correspondencia.

Recibido Dic. 14, 2018; Aceptado Feb. 25, 2019; Versión final Abr. 25, 2019, Publicado Oct. 2019

\begin{abstract}
Resumen
El presente artículo examina algunas diferencias de actitudes frente a las conductas deshonestas en estudiantes universitarios de las carreras de Administración y de Economía. El estudio es de alcance descriptivo, de corte transversal y de enfoque cuantitativo. Se administró un cuestionario a una muestra aleatoria de 275 estudiantes de la provincia del Guayas, Ecuador, durante el primer semestre del año 2017, donde se identificaron las actitudes deshonestas relacionadas con seis enunciados sobre el engaño en exámenes o evaluaciones escritas y las situaciones contextuales en que éstas surgen, agrupando los datos por carrera, género y el lugar de procedencia de los participantes. Se verificaron diferencias significativas entre grupos de variables mediante el análisis de tablas de contingencia empleando el estadístico chicuadrado. En el análisis entre grupos específicos, los hombres de la carrera de administración prefieren adoptar más actitudes deshonestas en comparación con estudiantes de economía. Los resultados entregan nuevos aportes a la literatura sobre la deshonestidad académica en el contexto latinoamericano.
\end{abstract}

Palabras clave: deshonestidad académica; engaño, estudiantes de pregrado; administración de empresas; economía; chi-cuadrado

\section{Differences in Attitudes towards Academic Dishonesty between Undergraduate Students of Administration and Economics in the Province of Guayas, Ecuador}

\begin{abstract}
This article examines some differences in attitudes towards dishonest behavior of economics and business undergraduate students. The study is of the cross-section type, with a descriptive scope and quantitative approach. A questionnaire was administered to a random sample of 275 students of the province of Guayas, Ecuador, during the first semester of 2017, where dishonest attitudes through six statements related to written test cheating were identified and the contextual situations in which they arise, grouping the data by career, gender and place of origin of the participants. Significant differences between groups of variables were verified through the analysis of contingency tables using the chi-square statistic. In the analysis between specific groups, male business students prefer to adopt more dishonest attitudes in comparison with students of economics. The results provide new contributions to the literature on academic dishonesty in the Latin American context.
\end{abstract}

Keywords: academic dishonesty; test cheating; undergraduate students; business administration; economy; chi-square 


\section{INTRODUCCIÓN}

El comportamiento humano crea muchas posturas con relación a la mentira y, de esta manera, el proceso de calificar o agrupar personas según su sinceridad es poco eficiente. La deshonestidad académica se ha convertido en una práctica común en instituciones educativas e investigaciones han identificado dicha temática como una potencial área de preocupación (Liebler, 2016; Sulphey y Jnaneswar, 2013). En vista de estos acontecimientos, el presente estudio aborda el fenómeno de deshonestidad académica en estudiantes universitarios. Bowers (1964) fue uno de los pioneros en tratar el tema de la deshonestidad en el ámbito académico, haciendo referencia al hecho de realizar actividades anti-éticas e ilegales o el uso de técnicas y formas de fraude que llevan a cabo los estudiantes en sus procesos de examen o evaluación de toda índole, con el propósito de mejorar sus calificaciones (Hollinger y Lanza, 2009; Hosny y Fatima, 2014). Inclusive, la globalización y aspectos relacionados con el uso generalizado del internet y dispositivos móviles ha hecho más fácil y cómoda la forma de cometer estos actos deshonestos (Friedman et al., 2016).

Varios tipos de deshonestidad académica han sido definidos por la literatura, como la colaboración al realizar tareas, la copia total o parcialmente la información de un compañero, el uso de internet como fuente de ayuda para resolver problemas complejos, presentación del mismo trabajo en diversos cursos o por distintas personas, uso de recursos ocultos durante un examen escrito, entre otros (Friedman et al., 2016). Es por esta razón que la deshonestidad académica se divide en tres principales categorías: (i) engaño o trampa, (ii) plagio y (iii) colusión. Las dos primeras son comunes de estudiantes jóvenes (Athanasou y Olasehinde, 2002; Liebler, 2016). En general, el engaño y el plagio tienen acciones similares al obtener ideas o material de otras fuentes, sin embargo, el plagio puede o no ser intencional por la falta de conocimiento de algunos estudiantes con respecto a normas pertinentes de citación y, por consiguiente, no siempre puede ser considerado como un engaño, lo que le otorga el tinte de ilegal (Carroll, 2002). El plagio es un tipo de violación de la integridad académica y ocurre cuando un individuo utiliza deliberadamente la redacción, las ideas u otros materiales originales de alguien más sin reconocer su origen (Quinn, 2006). La colusión por su parte se define como un convenio por medio del cual se pretende alcanzar cierto provecho a costa de un tercero, valiéndose de medios fraudulentos (Carroll, 2002).

La presente investigación se enfoca en la categoría de engaño desde la posición de la ética, como problema de deshonestidad académica, haciendo referencia al acto de copiar durante un examen o evaluación escrita. Se define así al engaño académico como una violación intencional de las reglas con la finalidad de obtener ventaja o mejores resultados en exámenes o formas similares de evaluación (Gedder, 2011). Esta conducta tiene repercusiones negativas en el aprendizaje, que conduce a la producción de graduados incompetentes con baja autoestima. Inclusive, estas acciones dañan la imagen de las instituciones educativas. (Farías y Pérez, 2010).

Existen varios métodos de engaño empleados por estudiantes universitarios. Usualmente, los estudiantes engañan por ignorancia con más frecuencia con relación a otras posibilidades existentes (Boehm, 2009). Esto muestra qué el avance académico estaría relacionado con la copia de manera indirecta. De esta manera se resumen los siguientes escenarios: (i) estudiantes que no tienen tiempo debido a la carga de otras actividades y (ii) estudiantes que tienen tiempo y no quieren estudiar (Broeckelman y Pollock, 2006). Ahmadi (2012) en un estudio realizado a 132 estudiantes iraníes determinó que los métodos más comunes son: (i) copiar de otros exámenes, (ii) hablar con compañeros adyacentes y (iii) usar gestos para obtener respuestas de otros. Según Hughes et al. (2006), los estudiantes frecuentemente asienten ante la posibilidad de ayudarse entre ellos. En el ambiente universitario, una persona llega a entablar relaciones con estudiantes de su carrera, facultad, universidad e incluso de otras universidades. Así, la premeditación crea un ambiente con mayores expectativas al momento de copiar, porque los implicados tenderán a conocer señales y códigos para el intercambio de información. A pesar de estas razones, varios hallazgos de autores que han abordado esta problemática desde el punto de vista ético han demostrado que los estudiantes conscientes de la dimensión del engaño académico no sólo mejoraron en pruebas sencillas, sino que también recomendaron penalidades más severas para delitos de deshonestidad académica (Fishman, 2016; Newton, 2016).

En términos de actitudes frente a la deshonestidad académica, diversas variables contribuyen en la decisión de copiar durante un examen. Demográficamente, la literatura avala que los estudiantes jóvenes son más propensos a indicar una elevada frecuencia de engaño académico que estudiantes de mayor edad (Liebler, 2016). Broeckelman y Pollock (2006) comparten esta noción. A través de encuestas en línea que se llevaron a cabo a estudiantes de pregrado y postgrado de Ohio University, se observó que el $84 \%$ de los estudiantes de pregrado y el $55 \%$ de los estudiantes de postgrado admiten que hicieron trampa o algún acto de engaño académico. Por tanto, los resultados exhiben una clara tendencia de que esos hábitos se reducen a medida que aumenta el nivel académico y conocimiento, junto con la edad del estudiante. 
Resultados de estudios previos también hacen énfasis en el programa de estudio al evaluar la deshonestidad académica (Josien et al., 2015). En un estudio sobre actitudes frente a diversos escenarios de engaño, Carpenter y Reimers (2005) observaron que estudiantes de programas de Negocios, Contabilidad e Ingeniería han sido señalados como estudiantes más propensos a mostrar conductas de deshonestidad académica que aquellos que provienen de carreras de humanidades y ciencias sociales. Esta conclusión es apoyada por estudios como el de Teixeira y Rocha (2010), quienes estimaron que la magnitud del engaño en acciones como copiar en un examen es mayor entre estudiantes de economía y de carreras administrativas y empresariales. Los resultados de la investigación indicaron que estos estudiantes tuvieron un $62 \%$ de representación en la muestra analizada. Simha et al. (2012) descubrieron que los estudiantes de negocios de Estados Unidos tenían actitudes mucho más relajadas hacia la trampa y que aparentemente parecen engañar más estudiantes de otras carreras.

Una de las características que ha atraído la atención de investigadores en la literatura referente a la deshonestidad académica es el género y los resultados son ambiguos. Nonis y Swift (2001) en su teoría de la socialización diferencial de los géneros, señalan que existen métodos diferenciados de enseñanza asociados al género de las personas y que, a partir de esta teoría, surge la posibilidad de encontrar diferencias entre actitudes, porque mientras que la educación de las niñas es conservadora, la de los niños tiene una apertura a realizar más cosas indebidas que las mujeres. Diversos estudios apoyan esta idea, señalando que las mujeres son menos propensas a caer en conductas de deshonestidad académica en comparación con las actitudes de los hombres ante estos escenarios (Kobayashi y Fukushima, 2012; Mensah et al., 2016). Por otro lado, Campos y Solano (2012) encontraron que, en mayor porcentaje, las mujeres han sido deshonestas, pero de manera ponderada deberían gestarse los datos por la superioridad que tienen en número con relación a los hombres en carreras de corte administrativo. Otros estudios también han reportado alta frecuencias de conductas deshonestas en las mujeres que en los hombres (DePalmer et al., 1995). En un estudio descriptivo sobre actitudes de deshonestidad académica en universitarios de carreras de administración, Sulphey y Jnaneswar (2013) encontraron que los hombres exhiben más comportamientos poco éticos que las mujeres. Nejati et al. (2011), mientras tanto, determinaron que no hay diferencia significativa del efecto entre géneros, coincidiendo con demás investigaciones que concluyeron la presencia de diferencias no significativas en la propensión del engaño académico entre mujeres y hombres (Ahmadi, 2012).

También existen casos muy diferenciados en torno al sector donde viven los estudiantes. Aquí la diferencia se puede dar por el ámbito cultural, asimismo por la característica religiosa en cada país. Bandura (1986) explica que el ejemplo marca una pauta al momento de las actitudes de las personas. De esta manera la estructura cultural de la sociedad va formando a las personas y, sin correcciones agresivas, seguirá perpetuándose de forma generacional. Usando como referencia la cultura religiosa, estudiantes de género femenino de origen saudí rechazan en un $65 \%$ las prácticas deshonestas guiadas por sus creencias en el Islam según Hosny y Fatima (2014). Boehm (2009) afirma que se emplean métodos adaptados a la posibilidad que se da en un momento determinando para escoger de qué manera copiar en un examen. Así la variación puede originarse por el tipo de aula donde se da el examen, el grado de amistad con el compañero que se sienta cerca, el tipo de profesor, la cantidad de estudiantes completando el examen escrito, entre otros.

En el contexto ecuatoriano, las probabilidades son condicionadas, debido a que los exámenes, en un número importante de universidades, constituyen el $50 \%$ de la nota de cada parcial y se necesita de un $70 \%$ en promedio para superar la barrera de la reprobación. Esto sugiere que las personas que no rindan lecciones y/o tutorías o proyectos (ambos se reparten el otro $50 \%$ de la nota equitativamente) tendrán más afinidad con actos deshonestos. Con base en estos factores previamente mencionados y con la revisión de literatura, el estudio pretendió encontrar diferencias sobre las actitudes de estudiantes universitarios de carreras de economía y de administración frente a diversas situaciones de deshonestidad académica, específicamente cuando otro estudiante está copiando en un examen. Estas carreras fueron seleccionadas debido a que sus estudiantes son considerados potencialmente como los futuros empresarios y líderes económicos y políticos del mañana, por lo que sus prácticas pueden afectar la definición de ética empresarial y economía aceptable. Además, la evaluación de este fenómeno se ha centrado casi exclusivamente en el contexto de los Estados Unidos (Teixeira y Rocha, 2010). Se espera además que los resultados permitan proponer recomendaciones sobre cómo confrontar este fenómeno de engaño académico y generar conciencia de estos actos en los estudiantes.

\section{METODOLOGÍA}

El presente estudio es cuantitativo, de corte transversal con fuente de datos primarios y de naturaleza descriptiva (Hernández et al., 2006). Para el desarrollo de la investigación, se distribuyó un cuestionario en forma de encuestas anónimas a través de un formato basado en una comparación de las actitudes hacia el engaño entre estudiantes estadounidenses e internacionales planteado por Wendy Bailey y Scott Bailey (2011). Dichos autores plantearon seis enunciados, de modo que cada uno medía la actitud de cada 
estudiante frente a un escenario de deshonestidad académica. Cada situación es planteada a los participantes a partir de la premisa "En caso de ver a un estudiante copiando durante el examen, yo...": (i) lo ignoro y sigo con mi resolución, (ii) le sugiero que deje de copiar, (iii) le informo al profesor de forma anónima, (iv) le informo al profesor de forma directa, (v) le informo a una autoridad superior y (vi) le pido ayuda con mi resolución. Sin embargo, con el fin de ajustar la traducción y redacción de los enunciados a las necesidades de la investigación actual, se realizó una prueba piloto. Cabe destacar que se eliminaron términos poco familiares para los estudiantes y se establecieron enunciados simples y concisos para minimizar ambigüedad en el cuestionario. Los resultados de la prueba piloto permitieron determinar que el método de recolección de datos fue apropiado y que los enunciados fueron de fácil entendimiento, lo que brinda validez de contenido.

La población del estudio estuvo conformada por estudiantes matriculados de dos universidades públicas de la provincia del Guayas, que cursaban las carreras de Administración de Empresas y de Economía. De acuerdo con información del Sistema Nacional de Información de Educación Superior del Ecuador (SNIESE, 2017), en el Guayas existen tres universidades públicas que ofertan estas carreras y albergan aproximadamente 85.000 estudiantes, que representan el $20 \%$ de estudiantes matriculados en el sistema público nacional. La muestra final del estudio estuvo conformada por 275 cuestionarios válidos de estudiantes que cursaban las carreras de Administración de Empresas y de Economía y los datos fueron levantados durante el primer semestre del año 2017. La modalidad de encuesta anónima permitió llegar más a fondo de la realidad de cada estudiante y que esto genere menos error al momento del cálculo. De igual modo, se clasifica a la modalidad de estudios en tres niveles: (i) Básico, (ii) Intermedio y (iii) Avanzado. Así, cada nivel comprende tres ciclos de pregrado y, por lo general, en el último nivel se dan solo dos ciclos, porque la mayoría de carreras ofertan ocho semestres.

Para cada enunciado se utilizaron escalas de Likert de cinco puntos, tal como la investigación original de Bailey y Bailey (2011), cuyas opciones de respuesta son: (i) muy en desacuerdo, (ii) en desacuerdo, (iii) ni de acuerdo ni en desacuerdo, (iv) de acuerdo y (v) muy de acuerdo. Para algunos grupos, las frecuencias observadas fueron bastante bajas en ciertas categorías de respuestas. Por ello, se recodificaron las respuestas de cada enunciado a tres escalas en: (i) de acuerdo, (ii) ni de acuerdo ni en desacuerdo, y (iii) en desacuerdo; para satisfacer el supuesto de la muestra general (Tabla 1).

Tabla 1: Descripción de enunciados del estudio y su recategorización

\begin{tabular}{|c|c|c|c|c|}
\hline Enunciado & Descripción & \multicolumn{3}{|c|}{ Opciones de respuesta } \\
\hline $\begin{array}{l}\text { Enunciado } \\
1\end{array}$ & $\begin{array}{l}\text { "En caso de ver a un estudiante copiando } \\
\text { durante un examen, lo ignoro y sigo con mi } \\
\text { resolución" }\end{array}$ & (1) En desacuerdo & $\begin{array}{l}\text { (2) Ni de acuerdo } \\
\text { ni en desacuerdo }\end{array}$ & (3) De acuerdo \\
\hline $\begin{array}{l}\text { Enunciado } \\
2\end{array}$ & $\begin{array}{l}\text { "En caso de ver a un estudiante copiando } \\
\text { durante un examen, le sugiero que deje de } \\
\text { copiar" }\end{array}$ & (1) En desacuerdo & $\begin{array}{l}\text { (2) Ni de acuerdo } \\
\text { ni en desacuerdo }\end{array}$ & (3) De acuerdo \\
\hline $\begin{array}{l}\text { Enunciado } \\
3\end{array}$ & $\begin{array}{l}\text { "En caso de ver a un estudiante copiando } \\
\text { durante un examen, le informo al profesor de } \\
\text { forma anónima" }\end{array}$ & (1) En desacuerdo & $\begin{array}{l}\text { (2) Ni de acuerdo } \\
\text { ni en desacuerdo }\end{array}$ & (3) De acuerdo \\
\hline $\begin{array}{l}\text { Enunciado } \\
4\end{array}$ & $\begin{array}{l}\text { "En caso de ver a un estudiante copiando } \\
\text { durante un examen, le informo al profesor de } \\
\text { forma directa" }\end{array}$ & (1) En desacuerdo & $\begin{array}{l}\text { (2) Ni de acuerdo } \\
\text { ni en desacuerdo }\end{array}$ & (3) De acuerdo \\
\hline $\begin{array}{l}\text { Enunciado } \\
5\end{array}$ & $\begin{array}{l}\text { "En caso de ver a un estudiante copiando } \\
\text { durante un examen, le informo a una } \\
\text { autoridad superior" }\end{array}$ & (1) En desacuerdo & $\begin{array}{l}\text { (2) Ni de acuerdo } \\
\text { ni en desacuerdo }\end{array}$ & (3) De acuerdo \\
\hline $\begin{array}{l}\text { Enunciado } \\
6\end{array}$ & $\begin{array}{l}\text { "En caso de ver a un estudiante copiando } \\
\text { durante un examen, le pido ayuda con mi } \\
\text { resolución" }\end{array}$ & (1) En desacuerdo & $\begin{array}{l}\text { (2) Ni de acuerdo } \\
\text { ni en desacuerdo }\end{array}$ & (3) De acuerdo \\
\hline
\end{tabular}

Se realizó un análisis de tablas de contingencia por medio del estadístico chi-cuadrado para determinar si existe relación entre cada grupo de variables y la opción de respuesta frente a la situación planteada. En este sentido, la tabla 2 ilustra la estructura del análisis por variable y opción de respuesta de cada enunciado. Por tanto, el análisis se desarrolló en tablas de contingencia de: (i) el género frente a cada opción de respuesta, (ii) la procedencia frente a cada opción de respuesta y (iii) la carrera frente a cada opción de respuesta. Posteriormente se examinaron grupos específicos: (i) entre mujeres, por la carrera frente a cada opción de respuesta y por la procedencia frente a cada opción de respuesta, (ii) entre hombres, por la carrera frente a cada opción de respuesta y por la procedencia frente a cada opción de respuesta, (iii) entre guayasenses, por el género frente a cada opción de respuesta y por la carrera frente a cada opción de respuesta, (iv) entre no guayasenses, por el género frente a cada opción de respuesta y por la carrera frente a cada opción de respuesta, (v) entre estudiantes de administración, por el género frente a cada opción de respuesta y por la procedencia frente a cada opción de respuesta y (vi) entre estudiantes de economía, por el género frente a cada opción de respuesta y por la procedencia frente a cada opción de respuesta. 
Tabla 2: Estructura del análisis por cada opción de respuesta y categorización de variables

\begin{tabular}{|l|c|c|}
\hline \multicolumn{1}{|c|}{ Grupos } & Variable 1 & Variable 2 \\
\hline Todos & Género & Opción de respuesta \\
Todos & Procedencia & Opción de respuesta \\
Todos & Carrera & Opción de respuesta \\
Femenino & Carrera & Opción de respuesta \\
Femenino & Procedencia & Opción de respuesta \\
Masculino & Carrera & Opción de respuesta \\
Masculino & Procedencia & Opción de respuesta \\
Guayasenses & Género & Opción de respuesta \\
Guayasenses & Carrera & Opción de respuesta \\
No Guayasenses & Género & Opción de respuesta \\
No Guayasenses & Carrera & Opción de respuesta \\
Administración & Género & Opción de respuesta \\
Administración & Procedencia & Opción de respuesta \\
Economía & Género & Opción de respuesta \\
Economía & Procedencia & Opción de respuesta \\
\hline
\end{tabular}

El programa estadístico SPSS versión 22 fue empleado para tabular y analizar la información obtenida a través de los cuestionarios administrados. Con el uso del estadístico chi cuadrado, con un nivel de confianza (NC) de $95 \%(\alpha=0.05)$ y 2 grados de libertad $(\mathrm{gl})$, la región de rechazo se ubica en la zona $\geq 5.991$. Se plantean así la hipótesis nula $\left(\mathrm{H}_{0}\right)$ y la hipótesis alternativa $\left(\mathrm{H}_{1}\right)$ por cada tabla de contingencia: (i) $\mathrm{H}_{0}$ : No existe diferencias significativas entre las opciones de respuestas de cada enunciado para el grupo de variables seleccionado, (ii) $\mathrm{H}_{1}$ : Existe diferencias significativas entre las opciones de respuestas de cada enunciado para el grupo de variables seleccionado.

\section{RESULTADOS}

La tabla 3 presenta los resultados de las estadísticas descriptivas de los 275 estudiantes. Se destaca que el $44 \%$ de los participantes son de género masculino y el $56 \%$ restante de género femenino. La media de las edades es de 20 años, con una desviación estándar de 2.25 años. La edad con mayor frecuencia fue 19 años. Un $73.45 \%$ de los estudiantes son de la provincia del Guayas y el $26.55 \%$ restante provienen de otras provincias del Ecuador. Se observa además que el $71.27 \%$ de los participantes son de la carrera de Administración de empresas y el $28.73 \%$ restante pertenece a la carrera de Economía. El nivel de estudios muestra que un $27.64 \%$ cursan ciclos de nivel avanzado, un $40.36 \%$ de nivel intermedio y un $32 \%$ de nivel básico.

Tabla 3: Estadística descriptiva de las variables principales del estudio.

\begin{tabular}{|l|l|c|c|}
\hline \multicolumn{1}{|c|}{ Variable } & \multicolumn{1}{|c|}{ Medición } & Media o frecuencia & Porcentaje \\
\hline Edad & En años & 20 & - \\
& & $(2.25)$ & - \\
Género & (1) Masculino & 121 & $44.00 \%$ \\
& (2) Femenino & 154 & $56.00 \%$ \\
Procedencia & (1) Guayasenses & 202 & $73.45 \%$ \\
& (2) No Guayasenses & 73 & $26.55 \%$ \\
\multirow{5}{*}{ Nivel de estudios } & (1) Administración de empresas & 196 & $71.27 \%$ \\
& (2) Economía & 79 & $28.73 \%$ \\
& (1) Básico & 88 & $32.00 \%$ \\
& (2) Intermedio & 111 & $40.36 \%$ \\
& (3) Avanzado & 76 & $27.64 \%$ \\
\hline
\end{tabular}

El siguiente análisis corresponde a los resultados por enunciado de acuerdo a la variable género, que tiene como finalidad determinar relaciones entre el género y la actitud de los estudiantes frente a actitudes deshonestas como copiar durante un examen (Tabla 4). Frente al primer enunciado "En caso de ver a un estudiante copiando durante un examen, yo lo ignoro y sigo con mi resolución", se evidencia la tendencia a ignorar al compañero que copia, representado en un $68 \%$ de los participantes que respondió a la opción de acuerdo. Con respecto al enunciado 2 "En caso de ver a un estudiante copiando durante un examen, yo le sugiero que deje de copiar" y enunciado 6 "En caso de ver a un estudiante copiando durante un examen, le 
pido ayuda con mi resolución", las respuestas demuestran seguir una tendencia neutral con el $57.82 \%$ y $53.09 \%$ de los casos, respectivamente. El enunciado 4 "En caso de ver a un estudiante copiando durante un examen, le informo al profesor de forma directa" y enunciado 5 "En caso de ver a un estudiante copiando durante un examen, le informo a una autoridad superior" muestran una clara tendencia de desacuerdo como indican a las respuestas, con el $60 \%$ y $57.5 \%$ respectivamente. El mismo caso ocurre con el enunciado 3 "En caso de ver a un estudiante copiando durante un examen, le informo al profesor de forma anónima", sin embargo, existe una mínima diferencia con respecto a la opción de respuesta neutral.

Dado un nivel de confianza de $95 \%$ y dos grados de libertad, se verifica solamente una hipótesis alternativa $\mathrm{H}_{1}$ : existe diferencias significativas entre el género de los estudiantes participantes para el enunciado seis "En caso de ver a un estudiante copiando durante un examen, le pido ayuda con mi resolución" (9.2535 > 5.991). Este antecedente concluye que las mujeres tienden a presentar actitudes más deshonestas que los hombres a la hora de pedir ayuda para resolver el examen cuando el compañero está copiando. Las mujeres contestaron estar de acuerdo con un $34.17 \%$ versus los hombres con un $30.32 \%$. Por otro lado, los hombres contestaron estar en desacuerdo en un $20.65 \%$, ampliamente superior a las mujeres con un $7.5 \%$

Tabla 4: Resultado de la prueba chi-cuadrado por enunciado de acuerdo a la variable género

\begin{tabular}{|c|c|c|c|c|c|c|}
\hline \multirow{2}{*}{\multicolumn{2}{|c|}{ Características }} & \multicolumn{3}{|c|}{ Opción de respuesta } & \multirow{3}{*}{$\begin{array}{c}\text { Total } \\
155\end{array}$} & \multirow{3}{*}{$\begin{array}{c}x^{2} \\
0.1326\end{array}$} \\
\hline & & \multirow{4}{*}{$\begin{array}{c}\text { De acuerdo } \\
105 \\
82 \\
187\end{array}$} & \multirow{4}{*}{$\begin{array}{c}\begin{array}{c}\text { Ni de acuerdo ni en } \\
\text { desacuerdo }\end{array} \\
47 \\
35 \\
82\end{array}$} & \multirow{4}{*}{$\begin{array}{c}\text { En desacuerdo } \\
3 \\
3 \\
6\end{array}$} & & \\
\hline Enunciado 1 & Masculino & & & & & \\
\hline & Femenino & & & & 120 & \\
\hline & Total & & & & 275 & \\
\hline \multirow[t]{3}{*}{ Enunciado 2} & Masculino & 22 & 97 & 36 & 155 & 3.3034 \\
\hline & Femenino & 22 & 62 & 36 & 120 & \\
\hline & Total & 44 & 159 & 72 & 275 & \\
\hline \multirow[t]{3}{*}{ Enunciado 3} & Masculino & 12 & 61 & 82 & 155 & 0.2295 \\
\hline & Femenino & 10 & 50 & 60 & 120 & \\
\hline & Total & 22 & 111 & 142 & 275 & \\
\hline \multirow[t]{3}{*}{ Enunciado 4} & Masculino & 6 & 50 & 99 & 155 & 2.2835 \\
\hline & Femenino & 5 & 49 & 66 & 120 & \\
\hline & Total & 11 & 99 & 165 & 275 & \\
\hline \multirow[t]{3}{*}{ Enunciado 5} & Masculino & 8 & 49 & 98 & 155 & 4.8524 \\
\hline & Femenino & 8 & 52 & 60 & 120 & \\
\hline & Total & 16 & 101 & 158 & 275 & \\
\hline \multirow[t]{3}{*}{ Enunciado 6} & Masculino & 47 & 76 & 32 & 155 & 9.2535 \\
\hline & Femenino & 41 & 70 & 9 & 120 & \\
\hline & Total & 88 & 146 & 41 & 275 & \\
\hline
\end{tabular}

La tabla 5 detalla los resultados por enunciado de acuerdo a la variable procedencia, que tiene como finalidad determinar relaciones entre el lugar de procedencia y la actitud de los estudiantes frente a actitudes deshonestas como copiar durante un examen. En respuesta al primer enunciado "En caso de ver a un estudiante copiando durante un examen, yo lo ignoro y sigo con mi resolución", la mayoría de los estudiantes indicaron estar en de acuerdo en ignorar la situación con un $68 \%$ y no parece existir diferencia significativa basada en el lugar de procedencia. Con respecto al segundo enunciado donde se pregunta al estudiante si sugeriría a la persona que deje de copiar, el 59.27\% indicó que mantendría una actitud neutral frente a esta situación, seguido del $25.82 \%$ que está en desacuerdo. La misma tendencia ocurre con el enunciado 6 "En caso de ver a un estudiante copiando durante un examen, le pido ayuda con mi resolución". La mayoría de los participantes manifestaron una actitud neutral con un 53.09\%, sin embargo, un $32.36 \%$ respondió estar de acuerdo en solicitar ayuda a la persona que está copiando en el examen.

Los enunciados 3, 4 y 5 presentan resultados mayoritariamente en desacuerdo. En este sentido, los estudiantes indicaron que están en desacuerdo en informar al profesor de forma anónima la conducta deshonesta del compañero que está copiado con un 51.64\%, en informar al profesor de forma directa con un $59.27 \%$ e informar a una autoridad superior con un $57.45 \%$. A todas las tres situaciones les sigue una actitud neutral, con un $40.73 \%, 36.36 \%$ y $36.73 \%$ respectivamente. De acuerdo a un nivel de confianza de $95 \%$ y dos grados de libertad, el enunciado 2 presenta diferencias estadísticamente significativas basado en el lugar de procedencia $(8.8378>5.991)$. Se verifica así que las personas que provienen de otras provincias del Ecuador prefieren adoptar en su mayoría una actitud neutral (74.29\%) y muy pocas evidencian estar de 
acuerdo o en desacuerdo en sugerir al compañero que deje de copiar (8.57\% y $17.14 \%$ respectivamente). Por otro lado, los guayasenses también prefieren mostrar una actitud neutral (54.15\%) pero existen mayores grados de desacuerdo en sugerir al compañero que evite copiar en el examen (28.78\%).

Tabla 5: Resultado de la prueba chi-cuadrado por enunciado de acuerdo a la variable procedencia

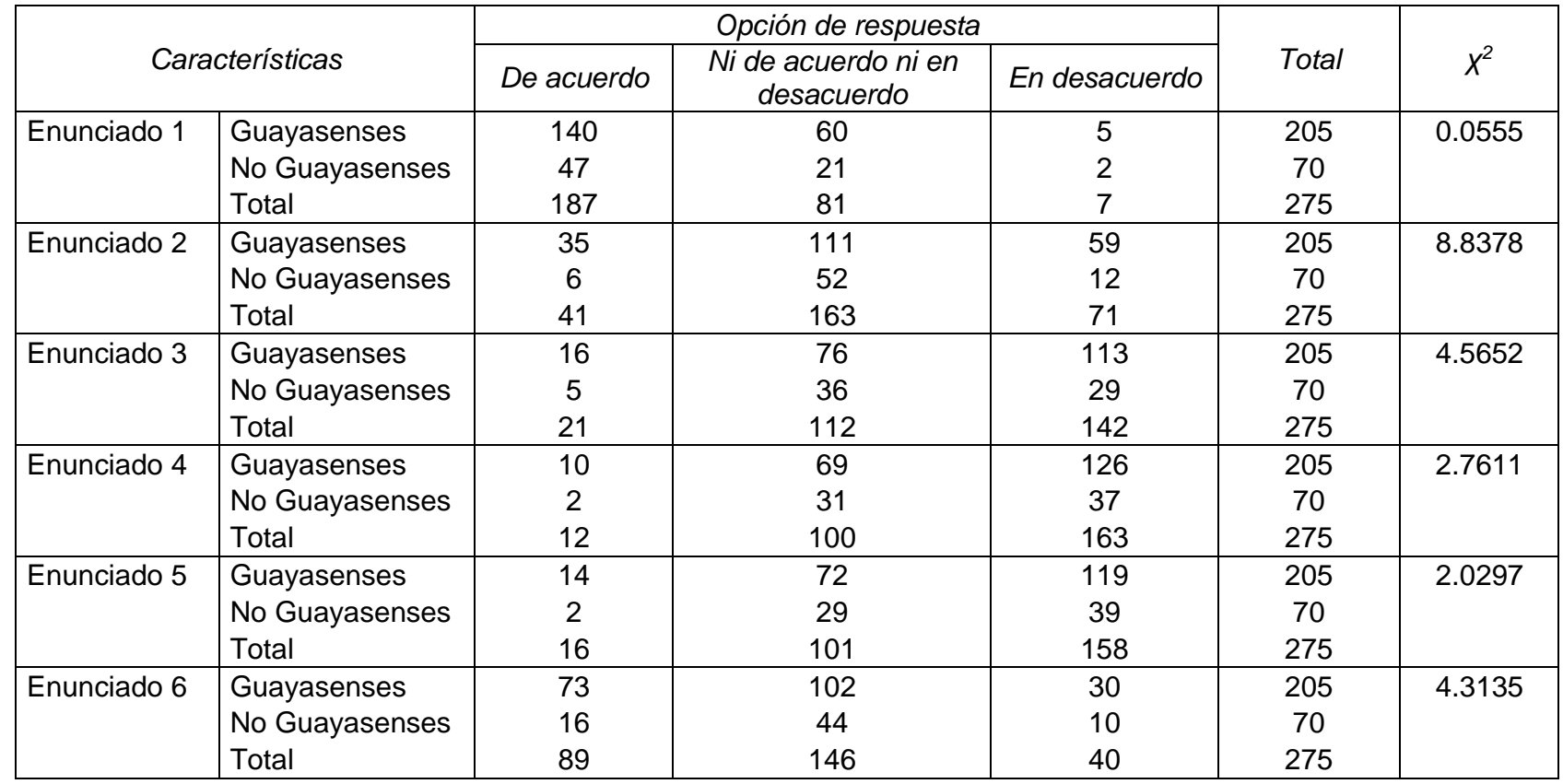

En siguientes análisis, no se apreciaron diferencias significativas entre las carreras de administración y de economía para cada enunciado del estudio. El análisis continuó en una siguiente etapa donde se examinaron grupos específicos de variables. La tabla 6 a continuación exhibe dos escenarios donde las diferencias entre grupos fueron significativas $\left(X^{2}>5.991\right)$ : (i) con personas de género femenino entre carreras para el enunciado 1 "En caso de ver a un estudiante copiando durante un examen, yo lo ignoro y sigo con mi resolución"; y (ii) con personas de género masculino entre carreras para el enunciado 6 "En caso de ver a un estudiante copiando durante un examen, le pido ayuda con mi resolución".

Tabla 6: Resultado de la prueba chi-cuadrado por enunciado de acuerdo a la variable carrera

\begin{tabular}{|c|c|c|c|c|c|c|}
\hline \multirow{2}{*}{\multicolumn{2}{|c|}{ Características }} & \multicolumn{3}{|c|}{ Opción de respuesta } & \multirow[b]{2}{*}{ Total } & \multirow[b]{2}{*}{$x^{2}$} \\
\hline & & De acuerdo & $\begin{array}{c}\text { Ni de acuerdo ni en } \\
\text { desacuerdo }\end{array}$ & En desacuerdo & & \\
\hline $\begin{array}{l}\text { Género Feme } \\
\text { Enunciado } 1\end{array}$ & $\begin{array}{l}\text { ino } \\
\text { Administración } \\
\text { Economía } \\
\text { Total }\end{array}$ & $\begin{array}{c}86 \\
20 \\
106\end{array}$ & $\begin{array}{l}27 \\
20 \\
47\end{array}$ & $\begin{array}{l}0 \\
2 \\
2\end{array}$ & $\begin{array}{c}113 \\
42 \\
155\end{array}$ & 13.0495 \\
\hline $\begin{array}{l}\text { Género Mascl } \\
\text { Enunciado } 6\end{array}$ & $\begin{array}{l}\text { ino } \\
\text { Administración } \\
\text { Economía } \\
\text { Total }\end{array}$ & $\begin{array}{l}30 \\
11 \\
41 \\
\end{array}$ & $\begin{array}{l}46 \\
20 \\
66 \\
\end{array}$ & $\begin{array}{c}6 \\
7 \\
13 \\
\end{array}$ & $\begin{array}{c}82 \\
38 \\
120 \\
\end{array}$ & 10.9925 \\
\hline
\end{tabular}

Los resultados brindan evidencias que las estudiantes mujeres inscritas en la carrera de administración están de acuerdo en ignorar la situación y continuar con su examen mientras que las de la carrera de economía prefieren tomar una actitud neutral. Con respecto a los estudiantes varones, se constata que los de la carrera de administración presentan actitudes más deshonestas, indicando estar de acuerdo en solicitar ayuda al compañero que está copiando (36.59\%) versus los estudiantes de economía (28.95\%). Finalmente, no se apreciaron diferencias significativas para las demás combinaciones de grupos y los enunciados. En síntesis, los resultados evidenciaron diferencias de las actitudes de los estudiantes de pregrado frente a una situación de deshonestidad académica en un examen. A modo general, existe una tendencia mayoritaria a ignorar al compañero que copia en una prueba y simplemente seguir con la evaluación. Esta tendencia se mantiene al analizar las respuestas por género, lugar de procedencia y por carrera.

En términos de abordar una acción explícita a tomar de acuerdo a cada situación, la mayoría de los estudiantes optaron por una actitud neutra en confrontar al compañero directamente, sugiriéndole que deje de copiar o solicitándole ayuda para el propio examen. No obstante, mostraron desacuerdo en reportar la 
acción no ética del compañero en todas las formas indicadas, sin distinción de género, procedencia o carrera universitaria en curso. Esto difiere de otros estudios que expresan que los estudiantes prefieren confrontar al estudiante directamente antes de reportar la acción de forma anónima o personal al instructor (Bailey y Bailey, 2011). Parece demostrarse así que la discrepancia entre resultados se debe al factor de la cultura.

Con respecto al género, se constataron diferencias significativas en una situación de solicitar ayuda al compañero que está copiando en el examen. En este sentido, las mujeres presentaron, proporcionalmente hablando, actitudes ligeramente más deshonestas que los hombres al estar de acuerdo en pedir ayuda. Sin embargo, en el análisis por grupos específicos, se encontró que los hombres presentan actitudes más deshonestas dependiendo de la carrera que cursan al solicitar ayuda para la resolución de la prueba, mientras que las mujeres optan por simplemente ignorar la actitud indecente. Estos resultados parecen estar de acuerdo con estudios que demuestran que las mujeres están menos dispuestas a tolerar comportamientos poco éticos que los hombres (Athanasou y Olasehinde, 2002; Kobayashi y Fukushima, 2012; Mensah et al., 2016; Sulphey y Jnaneswar, 2013) y que la profesión o el área de interés en el que el estudiante se desenvuelve también incide en la aparición de conductas deshonestas (Josien et al., 2015). Los hallazgos del estudio ofrecen varias implicancias, sobre todo para instituciones universitarias y entes reguladores de aspectos relacionados con la educación. Bajo este contexto, las autoridades de estas organizaciones pueden tomar decisiones más allá del contenido de una cátedra en particular, considerando variables como la carrera que cursa el estudiante, el género y el lugar de procedencia. Factores como la enseñanza en principios, valores y ética desde la óptica académica pueden ser adoptados para generar conciencia de que el daño que provoca este tipo de acciones deshonestas afecta en mayor medida a la reputación y aprendizaje del mismo estudiante y reducir o eliminar estos hábitos.

Futuras investigaciones pueden ser desarrolladas a partir del presente estudio. Así, el procedimiento de evaluación de las actitudes deshonestas de los estudiantes se puede replicar de tal manera que se sea posible comparar facultades, universidades, empresas, entre otros agentes. El enfoque para los tipos de carrera también es importante, como por ejemplo una carrera de corte cualitativo contra una cuantitativa. De igual modo, sería importante enriquecer el análisis de estas situaciones considerando nuevos grupos de variables representativas que permita una evaluación más a fondo.

\section{CONCLUSIONES}

Las siguientes conclusiones surgen a partir de los hallazgos de la presente investigación: 1. Proporcionalmente, las estudiantes mujeres de universidades en Guayas evidenciaron estar mayormente de acuerdo que los hombres en solicitar ayuda al compañero que copia para resolver el examen; 2 . Frente al enunciado de sugerir al compañero que deje de copiar en una evaluación, los estudiantes que provienen de otras provincias del Ecuador mostraron una actitud neutral, es decir ni a favor ni en contra, en relación a los estudiantes de la provincia del Guayas, que indicaron estar en desacuerdo, de forma que la zona de procedencia también guarda relación con las actitudes de deshonestidad académica; 3 . El análisis entre grupos específicos determinó que las mujeres de la carrera de administración están de acuerdo en ignorar la situación y continuar con su examen, mientras que los hombres de la carrera de administración prefieren adoptar actitudes deshonestas como solicitar ayuda al compañero que está copiando, en comparación con estudiantes de economía; y 4. Esta investigación contribuye a la literatura acerca de actitudes académicas deshonestas, específicamente en la acción de hacer trampa en un examen o evaluación escrita. En este sentido, el estudio también reveló que el campo de acción profesional del estudiante y la zona de procedencia son importantes en la presencia de dichas actitudes.

\section{REFERENCIAS}

Ahmadi, A., Cheating on exams in the Iranian EFL context, Journal of Academic Ethics, 10, 151-170 (2012)

Athanasou, J. y O. Olasehinde, Male and female differences in self-report cheating, Practical Assessment, Research, \& Evaluation, 8(5), 1-12 (2002)

Bailey, W. y S. Bailey, A comparison of attitudes towards cheating between U.S. and international students, International Journal of Business Research, 11(5), 26-37 (2011)

Bandura, A., Social foundations of thought and action, Englewood Cliffs, Prentice Hall (1986)

Boehm, P., M. Justice y S. Weeks, Promoting Academic Integrity in Higher Education, The Community College Enterprise, 45-61 (2009)

Bowers, W.J., Student Dishonesty and Its Control in College, New York, Bureau of Applied Social Research, Columbia University (1964)

Broeckelman, M. y T. Pollock, An Honest Look at Academic Dishonesty, School of Communication Studies, Ohio (2006) 
Campos, J., y W. Solano, Deshonestidad académica en estudiantes de una universidad de Costa Rica, América Central, Revista Electrónica Actualidades Investigativas en Educación, 12(2), 1-28 (2012)

Carpenter, T., y J. Reimers, Unethical and fraudulent financial reporting: Applying the theory of planned behavior, Journal of Business Ethics, 60(2), 115-129 (2005)

Carroll, J., A handbook for deterring plagiarism in higher education, Oxford, Oxford Centre for Staff and Learning Development (2002)

DePalmer, M., S. Madey y S. Bornschein, Individual differences and cheating behavior: Guilt and cheating in competitive situations, Personality and Individual Differences, 18, 761-769 (1995)

Farías, D., y J. Pérez, Motivación en la Enseñanza de las Matemáticas y Administración, Formación Universitaria, 3(6), 33-40 (2010)

Fishman, T., Academic integrity as an educational concept, concern, and movement in US institutions of higher learning. In Handbook of academic integrity, 7-21, Springer Nature, Pringer Science - Business Media Singapore (2016)

Friedman, A., I. Blau e Y. Eshet-Alkalai, Cheating and Feeling Honest: Committing and Punishing Analog versus Digital Academic Dishonesty Behaviors in Higher Education, Interdisciplinary Journal of e-Skills and Lifelong Learning, 12, 193205 (2016)

Gedder, K. A., Academic dishonesty among gifted and high achieving students, Academic Dishonesty, 34(2), 50-56 (2011) Hernández, R., C. Fernández y P. Baptista, Metodología de la Investigación. México D. F., Mc Graw Hill (2006)

Hollinger, R. y L. Lanza, Academic dishonesty and the perceived of countermeasures: An empirical survey of cheating at a major public university, NASPA Journal, 38(3), 587-602 (2009)

Hosny, M., y S. Fatima, Attitude of Students towards Cheating and Plagiarism: University Case Study, Journal of Applied Sciences, 14(8), 748-757 (2014)

Hughes, J., y D. McCabe, Academic Misconduct within Higher Education in Canada, Canadian Journal of Higher Education, 36(2), 1 - 21 (2006)

Josien, L., E. Seeley, J. Csipak y R. Rampal, Cheating: Students and faculty's perception on potential cheating activity, Journal of Legal, Ethical and Regulatory Issues, 18, 21-38 (2015)

Kobayashi, E., y M. Fukushima, Gender, social bond, and academic cheating in Japan, Sociological Inquiry, 82(2), 282304 (2012)

Liebler, R., Collecting and reporting self-reports of the number of times cheated, College Student Journal, 50(1), 95-101 (2016)

Lind, D., W. Marchal y S. Wathen, Estadística aplicada a los negocios y la economía, México: McGraw-Hill / Interamericana Editores (2012)

Mensah, C., E. Azila y M. Appietu, Examination cheating attitudes and intentions of students in a Ghanaian polytechnic, Journal of Teaching in Travel \& Tourism, 16, 1-19 (2016)

Nejati, M., S. Ismail y A. Shafaei, Students' Unethical Behaviour: Insights from an African Country. The Journal of Global Business and Management Research, 3(3), 276-295 (2011)

Newton, P., Academic integrity: A quantitative study of confidence and understanding in students at the start of their higher education, Assesment \& Evaluation in Higher Education, 41(3), 482-497 (2016)

Nonis, S., y C. Swift, An examination of the relationship between academic dishonesty and workplace dishonesty: A multicampus investigation, Journal of Education for Business, 77(2), 69-77 (2001)

Quinn, M. J., Ethics for the Information Age. Boston, MA, Pearson (2006)

Simha, A., J. Armstrong y J. Albert, Who Leads and Who Lags? A Comparison of Cheating Attitudes and Behaviors among Leadership and Business Students, Journal of Education for Business, 87, 316-324 (2012)

Sistema Nacional de Información de Educación Superior del Ecuador. Geoportal SNIESE. Obtenido de Oferta académica vigente en el Ecuador (2017)

Sulphey, M.M. y K. Jnaneswar, A study on the Academic dishonesty, Anomia and Unethical Behaviour among Business Graduates, The Journal of Contemporary Management Research, 8(2), 57-72 (2013)

Teixeira, A. y M. Rocha, Cheating by economics and business undergraduate students: an exploratory international assessment. Higher Education, 59, 663-701 (2010) 
